Z Gerontol Geriat 2021 · 54:429-430 https://doi.org/10.1007/s00391-021-01934-3

Angenommen: 8. Juni 2021

(c) Springer Medizin Verlag $\mathrm{GmbH}$, ein Teil von Springer Nature 2021

\title{
Geriatrie - Herausforderungen im klinischen Alltag
}

\author{
M. Gosch $\cdot$ K. Singler ${ }^{1,2} \cdot$ H. J. Heppner ${ }^{3}$ \\ ${ }^{1}$ Medizinische Klinik 2, Schwerpunkt Geriatrie, Klinikum Nürnberg, Paracelsus Medizinische \\ Privatuniversität Nürnberg, Nürnberg, Deutschland \\ ${ }^{2}$ Institut für Biomedizin des Alterns, Friedrich-Alexander-Universität Erlangen-Nürnberg, Nürnberg, \\ Deutschland \\ ${ }^{3}$ Klinik für Geriatrie, Helios Klinikum Schwelm, Schwelm, Deutschland
}

Der aktuelle Themenschwerpunkt widmet sich unserem klinischen Alltag mit seinen vielfältigen Herausforderungen. Wie könnte es besser gelingen, die vielseitigen und spannenden Aspekte der Geriatrie anhand von verschiedenen Fällen darzustellen? Aus den zahlreichen Einreichungen hat eine unabhängige Jury 12 Fälle ausgewählt. Die ausgewählten Beiträge durchliefen vor der Publikation einen Reviewprozess durch zwei weitere Gutachter.

Natürlich freut es uns, dass unsere Idee so gut angenommen wurde. Die Fälle bilden sehr gut das weite interdisziplinäre Spektrum der Geriatrie ab. Es reicht von der Inneren Medizin, über die Psychiatrie und Neurologie bis hin zu den chirurgischen Fächern. In vielen Fällen zeigen sich die spezifischen geriatrischen Ansatzpunkte in der Abklärung und Therapie sowie die hohe Vulnerabilität der älteren Patienten. Auch der Aspekt der atypischen Krankheitspräsentation findet sich in mehreren Beiträgen wieder.

\section{》) Kaum ein anderes Fach hat so viele Facetten wie die Geriatrie}

Mit diesem Themenschwerpunkt möchten wir ganz bewusst die jüngeren Kolleginnen und Kollegen ansprechen und aufzeigen, dass kaum ein anderes Fach so viele Facetten hat wie die Geriatrie. Leider wird gerade der "Lerneffekt" der Geriatrie von jungen Kolleginnen und Kollegen sowie Studierenden häufig unterschätzt und nicht gesehen. Wie in vielen anderen Fächern auch, müssen wir um den Nachwuchs werben. Neben der Vielseitigkeit hat die Geriatrie viele weitere attraktive Aspekte. Insbesondere das Arbeiten im Team und der kommunikative Aspekt seien hier hervorgehoben.

Wir wünschen Ihnen mit dem aktuellen Themenschwerpunkt eine spannende und lehrreiche Lektüre. Nutzen Sie die Fälle, um Kolleginnen und Kollegen von der Geriatrie zu überzeugen. Ganz herzlich bedanken wollen wir uns bei den Autorinnen und Autoren (auch bei jenen, die dieses Mal nicht zum Zug gekommen sind) sowie den weiteren Juroren (namentlich in alphabetischer Reihenfolge ohne Titel: Petra Benzinger, Marija Djukic, Peter Dovjak, Corinna Drebenstedt, Helmut Frohnhofen, Werner Hofmann, Bernhard Iglseder, Thomas Münzer, Rupert Püllen, Ulrich Thiem). Ohne die engagierte Teamarbeit wäre dieser Themenschwerpunkt nicht zustande gekommen.

Univ.-Prof. Dr. med. univ. M. Gosch Univ.-Prof. Dr. med. Hans Jürgen Heppner apl. Prof. Dr. med. Katrin Singler 


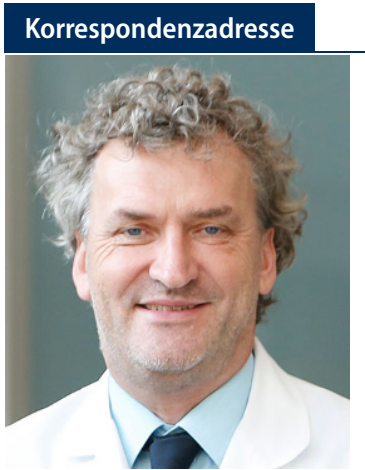

Univ.-Prof. Dr. med. univ. M. Gosch

Medizinische Klinik 2, Schwerpunkt Geriatrie, Klinikum Nürnberg, Paracelsus Medizinische Privatuniversität Nürnberg

Prof.-Ernst-Nathan-Str. 1, 90427 Nürnberg, Deutschland

Markus.Gosch@klinikum-nuernberg.de

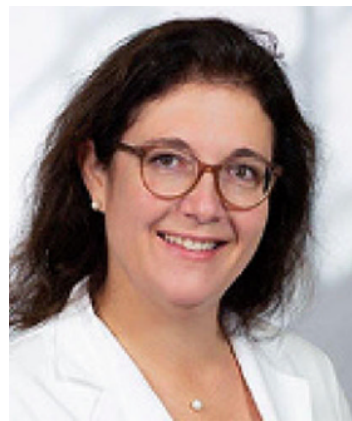

apl. Prof. Dr. med. K. Singler

Medizinische Klinik 2, Schwerpunkt Geriatrie, Klinikum Nürnberg, Paracelsus Medizinische Privatuniversität Nürnberg

Prof.-Ernst-Nathan-Str. 1, 90427 Nürnberg,

Deutschland

Katrin.Singler@klinikum-nuernberg.de

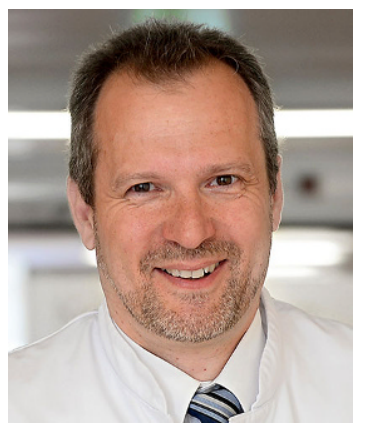

Univ.-Prof. Dr. med. H. J. Heppne

Klinik für Geriatrie, Helios Klinikum Schwelm Dr.-Moeller-Str. 15, 58332 Schwelm, Deutschland

Hans.Heppner@uni-wh.de

Interessenkonflikt. M. Gosch, K. Singler und H.J. Heppner geben an, dass kein Interessenkonflikt besteht.

\section{Suizidprävention für pflegebedürftige Menschen stärken}

Wenn Menschen suizidal sind, also etwa Suizidgedanken haben, kann das ganz unterschiedliche Gründe haben. Oft kommen mehrere Faktoren zusammen, die dazu beitragen. Ältere Menschen mit Hilfe- und Pflegebedarf leben häufig mit mehreren fortschreitenden Erkrankungen und verschiedenen psychischen sowie sozialen Belastungen. Zu solchen Belastungen gehören zum Beispiel stark nachlassende körperliche oder geistige Fähigkeiten, chronische Schmerzen, Angst vor zunehmender Hilfebedürftigkeit, sowie Verluste von Beziehungen oder mangelnde soziale Einbindung. Das Zusammenwirken solcher und anderer Belastungsfaktoren kann schließlich auch zu Lebensmüdigkeit bis hin zu Suizidalität führen. Für pflegende Angehörige kann es besonders schwierig sein, mit Suizidgedanken des pflegebedürftigen Menschen umzugehen. Sie sind teilweise über viele Jahre mit den Themen Sterben und Tod oder sogar Suizidalität bei dieser Person konfrontiert. Dabei gelten pflegende Angehörige ohnehin - insbesondere bei hoher Unterstützungsintensität - als psychisch überdurchschnittlich belastet.

Dr. Ralf Suhr, Vorstandsvorsitzender der Stiftung Zentrum für Qualität in der Pflege (ZQP), betont daher: „Lebensmüdigkeit und Suizidalität werden insgesamt noch zu wenig als relevante Themen im Zusammenhang mit Pflegebedürftigkeit wahrgenommen." Zu einer guten Unterstützung von pflegebedürftigen Menschen gehöre, dass Anzeichen für entsprechende Krisen von allen Akteuren im Versorgungsmix sowohl wahr- als auch ernstgenommen werden. Dies umfasse auch, physische und psychische Belastungen zu erkennen und therapeutisch so gut wie möglich zu reduzieren. Suhr erklärt weiter: „Häufig müssen sich gerade Angehörige mit der Lebensmüdigkeit oder Suizidalität eines pflegebedürftigen Lebenspartners oder Elternteils auseinandersetzen, Gespräche mit diesen dazu führen oder deren Verzweiflung aushalten. Auch in diesem Kontext sollten pflegende Angehörige daher stärker als Zielgruppe für entsprechende Entlastung und professionelle Unterstützung verstanden werden."

Um dazu beizutragen, dass diese Themen sowohl gesellschaftlich als auch im Gesundheitssystem verstärkt wahrgenommen werden sowie damit auch das Verständnis für die Bedeutung von Suizidprävention im Zusammenhang mit Pflegebedürftigkeit zu fördern, ist das ZQP der Allianz für Suizidprävention beigetreten und wird sich in konkreten Projekten entsprechend engagieren.

Die Teilnehmenden der Allianz unterstützen die Ziele des Nationalen Suizidpräventionsprogramms für Deutschland (NaSPro) und verbinden damit die Absicht, den Gedanken der Suizidprävention und suizidpräventive Maßnahmen in ihren jeweiligen Bereichen zu fördern. „Im NaSPro arbeiten Fachleute der Suizidprävention mit gesellschaftlichen Institutionen zusammen. Suizidprävention ist immer eine gesamtgesellschaftliche Aufgabe. Unterstützung und Hilfe in belastenden Lebenssituationen hat viele Gesichter und kann nur gemeinsam entwickelt und vorangebracht werden. Wir freuen uns über die kreative Zusammenarbeit mit dem ZQP, um pflegende Angehörige auch in existenziellen Notlagen zu unterstützen", sagt Prof. Dr. Reinhard Lindner, Leitung des NaSPro.

Mehr zum ZQP und seinen Arbeiten zum Themenkomplex Lebensende, Sterben und Tod im Zusammenhang mit Pflegebedürftigkeit oder gesundheitlichen Belastungen im hohen Alter finden Sie hier: www.zqp.de. Mehr zum NaSPro finden Sie hier: www.suizidpraevention.de.

Kontakt: Torben Lenz, email: torben.lenz@zqp.de 